\title{
Histopathological changes induced by Meloidogyne incognita in some ornamental plants
}

\begin{abstract}
Histopathological changes induced by the root-knot nematode (Meloidogyne incognita) in five ornamental plants, specifically, Calendula (Calendula officinalis), Centaurea (Centaurea montana), Papaver (Papaver somniferum), Chrysanthemum (Chrysanthemum morifolium) and Dianthus (Dianthus caryophyllus), were investigated. Based on the galling index (GI), Centaurea was classified as susceptible, Calendula as moderately susceptible, and Papaver as moderately resistant, while Chrysanthemum and Dianthus were highly resistant to $\mathrm{M}$. incognita infection. The histopathology of the galled roots of Centaurea, Calendula, and Papaver during later stages of infection showed that nematodes were localized entirely within the cortex and generally oriented horizontally to the vascular cylinder. Most of the females were mature, and a few of them were associated with egg masses. Giant cells with a variation in cell sizes were observed in the galled roots of all three of the plant species and exhibited a granular cytoplasm and hypertrophied nuclei as a typical reaction to nematode feeding.
\end{abstract}

Keyword: Histopathology; Gall index; Giant cell; Meloidogyne incognita; Nematode 